\title{
Modeling and Optimizing Wireless Body Area Network Data using PSO in Virtual Doctor Server
}

\author{
Pooja Mohnani \\ Research Scholar, \\ Jain University, \\ Associate Professor, \\ CMR Institute Of Technology Bangalore, \\ Life Member ISTE
}

\author{
Fathima Jabeen, PhD \\ Principal, \\ Islamiah Institute OfTechnology \\ Bangalore \\ Life Member ISTE, \\ Member IEEE, Fellow IEI
}

\begin{abstract}
Fostered interest in human health \& technological developments has increasingly paved way to (WBAN) Wireless body area network. With broader capabilities it focuses is to play a vital role for the improvement of human health. In this research paper, we propose to Conceptualize \& optimize medical data using PSO in virtual doctor server (VDS) in existing WBAN architecture. Existing architecture of WBAN consists of: wireless sensor, wireless actuator node, wireless central unit, a virtual doctor server (VDS) and wireless Personal Device (PD). Personal Digital Assistant (PDA) or smart phone can be used as PD. Based on the existing architecture mentioned above VDS will keep the historical data about the patient, optimize the data using PSO, generate advices, inform to take rescue action and can provide first aid assistance instructions on patient or any of his close relative's PDA's. VDS can further be used to improve security $\&$ develop potential solutions for secure network for WBAN system.
\end{abstract}

\section{General Terms}

Your general terms must be any term which can be used for general classification of the submitted material such as Pattern Recognition, Security, Algorithms et. al.

\section{Keywords}

WBAN, Server for Virtual doctor, Particle swarm optimization

\section{INTRODUCTION}

WBAN is a range limited frequency (RF) based wireless communication technology that creates a network of sensor in, on, or around human body. This is depicted in [1], the range of communication of these nodes/sensors approximates around $2 \mathrm{~m}$, and some of the other important specifications of WBAN are listed in Table 1. Wireless Body Area Network is a unique interconnection of nodes, shown in fig 1 is designed and developed for gathering the information of vital signs of the human body like body temperature, blood pressure, electrocardiograph etc. Health information can be monitored by using a variety of sensors that may either be integrated with clothing or can be on the human body or even under the human skin. Centrally located unit is responsible to establish communication between sensors and other communicating devices like cellular phone, PDA, Wi-Fi point in wireless manner. These devices communicate all information from the human body to the external world (Server for virtual doctor, emergency etc.)
Table 1: WBAN Specifications

\begin{tabular}{|c|c|}
\hline Characteristic & Distance range 0 $<5 \mathbf{5 m}$ \\
\hline Initiation time & $<80 \mathrm{~ns}$ \\
\hline Network connectivity time & $<1 \mathrm{sec} /$ device \\
\hline Consumed power & $\sim 1 \mathrm{~mW} / \mathrm{Mbps}$ \\
\hline No. of nodes /area & $2-4$ Nodes $/ \mathrm{m} 2$ \\
\hline Latency (backbone) & $10 \mathrm{~ms}$ \\
\hline No. of nodes & Max 100 devices $/$ Network \\
\hline
\end{tabular}

The low range, low power wireless personal network deployed nearest to the human body is called Wireless Body Area Network (WBAN) where each node/sensor placed can be uniquely identified and monitored.

\section{BACKGROUND}

This section introduces Wireless Body Area Network (WBAN) \& its Applications with insights to Particle swarm optimization.

\subsection{Wireless Body area network \& its Applications}

Use of wearable sensors can enable the necessary treatment at home for patients who suffered attack of diseases such as heart-attacks, sleep apnea, Parkinson disease and so on [3] [5]. Patients after treatment usually go through the recovery/rehabilitation process by following a strict routine.

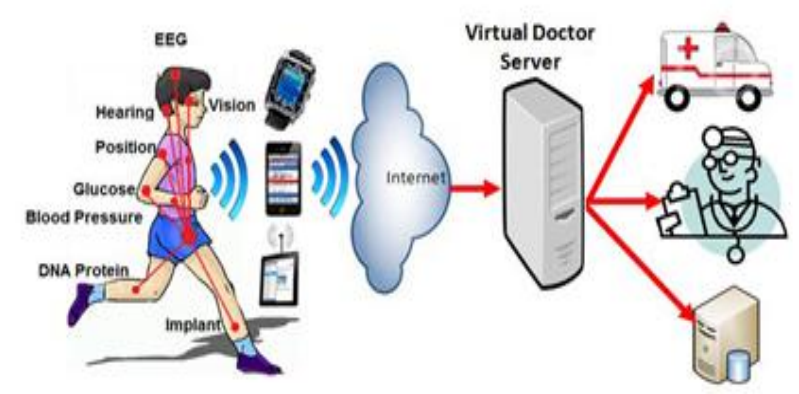

Figure 1 Architecture of WBAN

All the physiological signals as well as physical activities of the patient are possible to be monitored with the help of wearable sensors. During the rehabilitation stage the wearable sensors may provide audio feedback, virtual reality images and other rehabilitative services. The system can be tuned to the requirement [6] of individual patient. The whole activity can be monitored remotely by doctors, nurses or caregivers. 
Applications of WBAN are:

1) WBAN for monitoring the symptoms in the initial stage or (future) disease \& thus help in its intended treatment. Hence a variety of possibilities can be explored \& the disease can be prevented at a much earlier stage.

2) WBAN for professional athletes: WBAN can be helpful in tuning the training needs \& schedules of professional athletes.

3) WBAN for safety purposes in preventing medical accidents: Many lives are lost every year due to medical accidents, due to human error. Sensor Network can maintain a database and can notify the occurrence of similar accidents and thus can help in avoidance.

4) WBAN for uniformed personnel: by monitoring the level of toxics in the air for firefighters or soldiers an alarm or warning can help if life-threatening levels are detected.

\subsection{Particle Swarm Optimization}

Origin: It was proposed by Eberhart \& Kennedy in 1995, the Particle Swarm Optimization (PSO) algorithm was an outcome of the observations on foraging behavior of birds and their collective intelligence [16]. While foraging for food, a bird communicates and shares information with their other companions. Therefore, according to their personal best experiences and the swarm's best experiences, birds keep modifying their trajectory to find food for each individual as early as possible. Hence, the swarms of birds start their flight towards the same direction.

Analogous to Genetic Algorithm (GA), PSO algorithm can generally be used for optimization problems. Whilst, the difference between GA and PSO is, here (each particle in the PSO) has its own memory; it shares the information with neighbors then adjusts its behavior to align with the swarm's best experiences. These three features enable PSO to convergence rapidly and reach a good solution. Solution found by the PSO may not be the best, but definitely the closest solution after a number of iterations. Recently, the PSO algorithm has been applied to solve Traveling Salesman Problem, industrial load scheduling, routing optimization and complex non-linear optimization problems [7], [8]. The enormous amount of data obtained from the sensors put a huge burden on the system in terms of storage, analysis and future use.

The figure 2.1 shown below explains the flow of data optimization steps:

Step1: Initialize each particle with random positions $X_{i}^{0}$ and velocities $V_{i}^{0}$ in search space

Step2: Calculate $f\left(X_{i}^{0}\right)$, the fitness value of each particle, by fitness function and find out $\mathrm{P}_{\mathrm{i}}^{0}$, the local best, and $\mathrm{P}_{\mathrm{g}}^{0}$, the global best.

Step3: Search

Step4: Update $\mathrm{P}_{\mathrm{i}}^{\mathrm{k}}$ and $\mathrm{P}_{\mathrm{g}}^{\mathrm{k}}$

Step5: Update new position and velocity of each particle.
Step6: Determine maximum iteration number has been reached or stopped criteria have been met. If not go back to Step 3

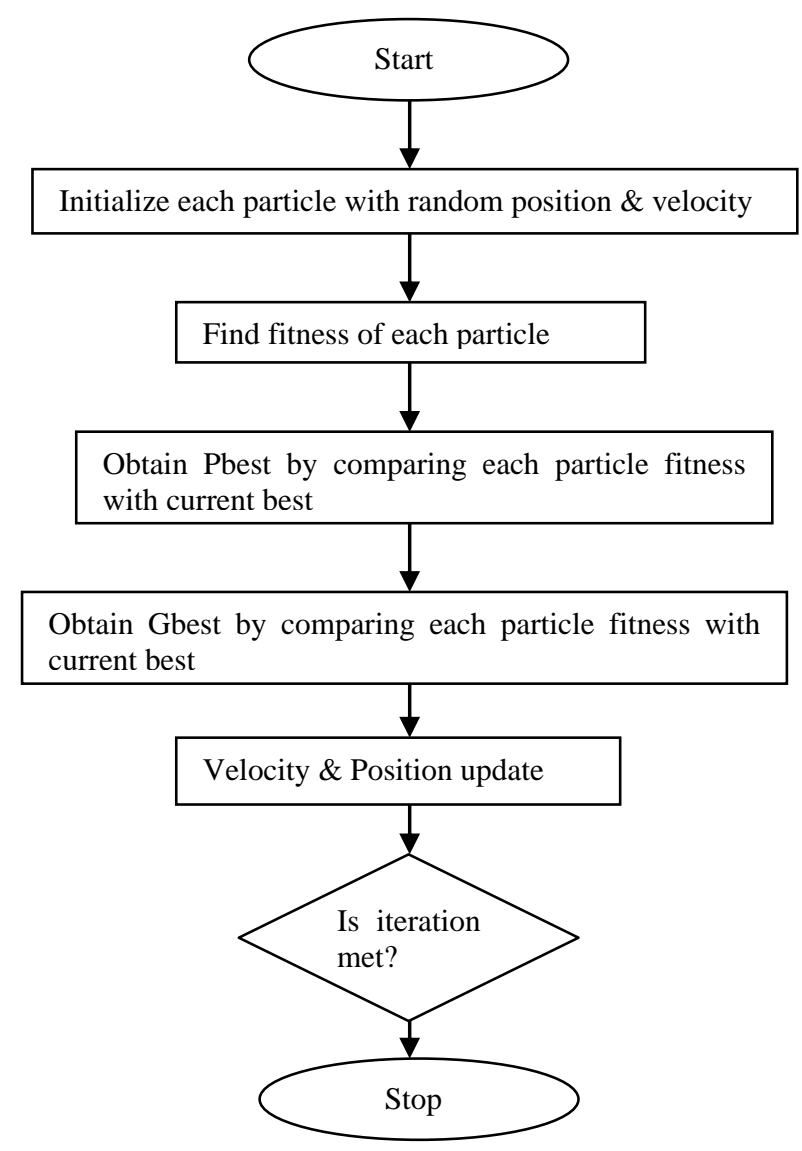

Figure 2.1 Flowchart of PSO

\subsection{Security}

The free- space utilization of the air media makes it prone for a malicious adversaries \& other security threats. Threats in wireless or wired medium are not uncommon $\&$ are a major point of concern today as most of the mobile computing environments are heterogeneous in nature. In wireless medium they can be improved by use of sophisticated algorithms. WBANs are made up of numerous small sized nodes that move with the body. This leads to hybrid network topology with varying RF energy absorption hence link quality, make routing a difficult task. This issue exists in nearly all the wireless networks, but is comparatively challenging in WBANs as the nature of network traffic is health-data, User specific and highly sensitive. Cryptographic security solutions are computation-intensive and more prone to data injection attacks under the assumption that body sensors have restricted resources. Data injection attack eats up the resources of the target network node by continuously transmitting the wrong data. For example, attacker may be intercepting the communication details of the target node, retrieve important information on authentication and use it to send wrong packets to the destination node. Without Know how of the situation, the target node puts intensive efforts to respond to the false packets; even worse, it then retransmit to other nodes. In energy-constrained WBANs, these attacks can consume sensor battery fast and reduce network lifetime. Sensors should be intelligent enough to recognize, reject \& 
notify false data at minimal cost. The misuse of data may create a huge problem on the acceptability of the system in the society so the necessary security issues need to be addressed in the design of embedded wearable sensors [22] as well as interoperability, connectivity and energy management should be taken care [23].

\section{DESIGN ISSUES IN WEARABLE SENSORS FOR HUMAN ACTIVITY MONITORING}

Research community is working hard to design and develop smart wearable devices to be used for continuous monitoring of different human activities. There are numerous challenges faced on design, customization, fabrication, deployment and utilization cum continuous monitoring. Designing challenges range from the hardware and software constraints due to form factor, size and low energy requirements, as well as there are safety issues such as avoidance of physical injury.

\section{- $\quad$ Power supply issues}

Physical operation needs an appropriate design of multiple sensor modules such as processor, radio, and optimization of data algorithm. WBAN devices are commonly powered by batteries, that may not be easy to interchange or replace, especially in cases where these devices are implanted inside the human body, then techniques like remote battery recharging are necessary. As the sensors are placed on the body, risk of thermal injury to tissue [8] need to be reduced by limiting the sensing frequency.

\section{- $\quad$ Element Constraints}

If the Sensor is needed to be implanted in the human body; then the shape, size and material to be used needs to be harmless to the body tissue. Furthermore, chemical reactions with body tissue and issues related to the disposal of the sensor are of extreme importance.

\section{- Durability}

Sensor network is implemented in varying environments; hence the probability of device failure is comparatively high. Designs need to have inbuilt mechanisms, alarms etc to handle failure, if there is failure in one node, it shouldn't cause the entire network's functionality. Uninterrupted operation therefore needs to be ensured for the biosensor during its lifetime.

\section{- $\quad$ Radio resource \& Network Management}

WBANs are uniquely deployed in mobile computing environments, operation management, sensor customization \& calibration in an context-aware performing network need to be updated as well. Sensor nodes may join/leave the network at any given time; this poses the requirements of configuring the devices on move. Strategies for management of resources, both for sensor functionalities and communication bandwidth, are critical.

\section{- $\quad$ Authorization \& Authentication issues}

Transfer of health-data between sensors in WBAN and over through network servers should be inherently secured; this data must be encrypted as to protect the patient's confidentiality \& hence integrity to the system. Medical practioner capturing this data must be ensured that the data is unique and originates from a given patient. [17], [18].Medical staff should neither be involved in establishment nor in authorization \& other related processes. Network resources must be available to the user as the same may not be capable of providing the password (i.e accessibility in emergency situations). Thus these processes must not consume major part of the available power resources \& be energy efficient \& reliable.

\section{- Quality of Service and Reliability}

According to [1], [20], [21] quality of service (QoS) handling is of prime importance in framing risk management of medical applications.A crucial issue, on reliability of the transmitted information in order to guarantee that the monitored data is received correctly by the health care professionals. The reliability can be considered either end-toend or on a per link base. Examples of reliability include the guaranteed delivery of data (i.e. packet delivery ratio), in order delivery. Moreover, messages should be delivered in reasonable time. The reliability of the network directly affects the quality of patient monitoring and in a worst-case scenario; it can be fatal when a life-threatening event has gone undetected.

\section{VIRTUAL DOCTOR SERVER MODEL \& OPTIMIZATION}

\subsection{Virtual Doctor Server}

The concept of virtual doctor server [7] integrates numerous situations of WBAN for management of the health related services and to provide help for health care service takers in emergency situation.

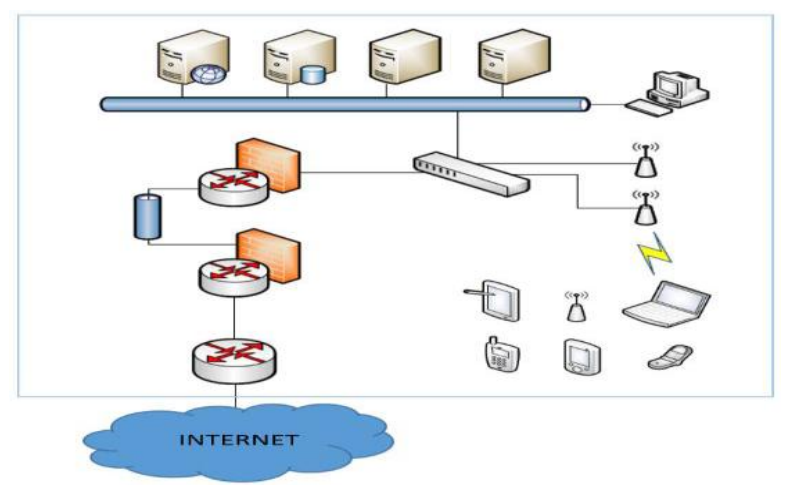

Figure 4.1 Virtual Doctor Server at Hospital

VDS can undertake the below mentioned:

- Maintain the database of patient and record the medication details of the patient and remind the same as suggested by doctor.

- Provide advice to the Patient on the basis of best practices and vital data collected.

- Inform ambulance or the emergency rescue team when required.

- $\quad$ Enable \& ensure Security, Confidentiality, Security and privacy of the patient data \& the underlying processes.

Patient can feed own condition using PD unit and vital data will be measured \& transmitted by the sensor, VDS can be configured on the basis of Patient's history stored in the 
database with the suggested best practices with the vital information. Suggestions are like:

- $\quad$ You are Fit

- Need rest, don't stress.

- Need first aid - take emergency medicine; No activity till doctor/paramedic will arrive etc.

\subsection{Particle Swarm Optimization}

As known in WBAN, the quantity of information is large because the data is real time data. In India doctor is to patient ratio is 1:5000, also with huge data per patient, there are issues related to the time performance and storage space. Hence, there is an urgent need for study \& finding new methods for detecting information from vital data. Design of a blood pressure [9] classification system in the WBAN environment is developed, which aims to forecast cardiac disorder which one with highest mortality amongst many others in India during these recent years. To understand \& process blood pressure data is stored in a database, for the same classification rules are created and the best rule is pointed depending on accuracy. Further, this rule is processes through the patient's data. Thus, Particle Swarm Optimization (PSO) Classification System in the WBAN is used to divide blood pressure data as normal and abnormal, as the first diagnosis before doctors can analyze. If patient's data is identified as abnormal, then the same will be sent to doctor for further action and get a second diagnosis. Thus, doctor can provide diagnosis to patients on priority basis \& can thus help in saving a life. Following shows a pictorial representation of the same:

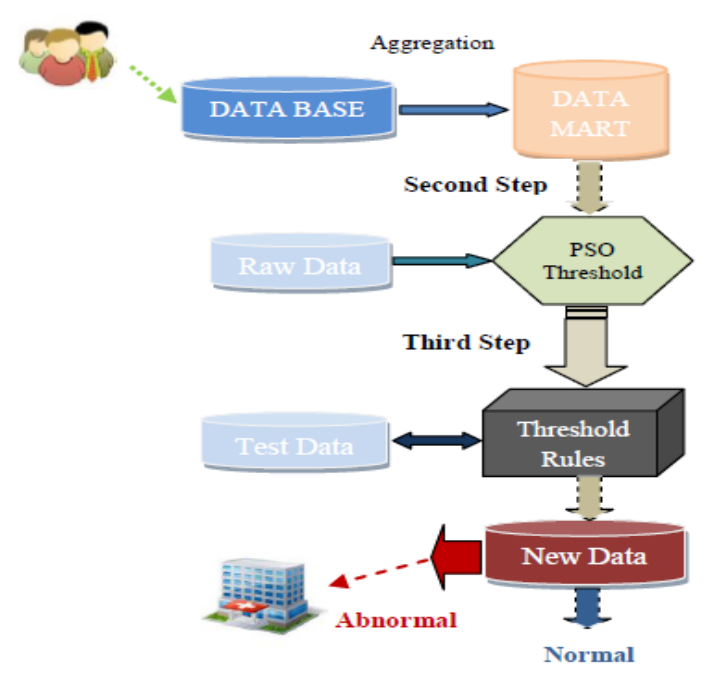

Figure 4.2 Data Classification system using PSO

\section{TODAY'S SCENARIO \& FUTURE OF WEARABLE DEVICES}

Development \& deployment of wearable devices has reached a stage where it can be used as normal household item, the high cost \& heterogeneity of the network is still holding it back. From deployment perspective, the prices of the product need to come down to a level so that people can afford them [10]. There is a huge market in a growing aging population \& patients suffering from chronic diseases which need monitoring in Asian countries along with the developed countries but the price has to be low. As per [10], wearable consumer devices such as fitness trackers, smart glasses and smart watches will be sold over 40 million in 2014 and is expected to be close to 100 million in 2015. The other challenge for wearable electronics for large scale deployment is to sustain the interest that it is with adequate functionality. The information from the wearable sensors may be extremely useful for long-term health monitoring and also in predicting the future health condition.

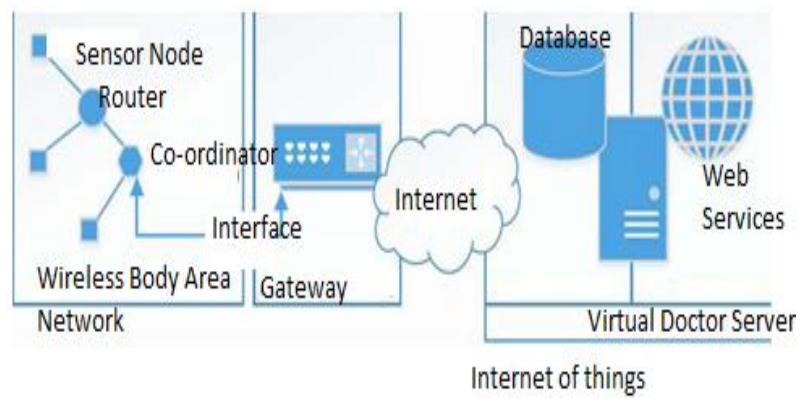

Figure 5.1 WBAN in mobile computing environment

The necessary block diagram representation in such a system is shown in figure 5.1. With broader dimension, this is a glimpse of hope that wearable device, Apple Watch etc, will be with us which will be similar to strapping a computer on our arm [11]. The watch is expected to be customized and is based on technology which attempts to colonize our bodies. The same is designed to track our movement, listen to our heartbeat and put our whole body on line. The data from the wearable devices may be used to determine the emotion of the person. Under monitoring too, an experimental platform as shown in fig 6 has been reported [12]. Or, may be the time will come when the whole computer may be fabricated in such a way that it will be possible for the human to wear it.

\section{CONCLUSION}

The paper has put forth the reported literature on wearable sensors and devices for monitoring human activities which aims to improve quality of life. The human activity monitoring is a vibrant area of research and a number of commercial developments are reported. Architecture of WBAN is integrated with the advantage of VDS \& data optimization using PSO. WBAN is an emerging technology aimed to immensely help the elderly, expecting mothers \& patients with chronic diseases. The challenges faced by the current design will also be addressed for future devices. Formal and Informal understanding predicts an increased interest, many more light-weight, high performance wearable devices with sophisticated systems for monitoring a wide range of activities.

\section{ACKNOWLEDGMENTS}

Thanks to Jain University for giving us the opportunity to work in this domain \& contribute.

\section{REFERENCES}

[1] B. Zhen, H.-bang Li, and R. Kohno,"Networking issues in medical implant communications," International Journal of Multimedia and Ubiquitous Engineering, vol. 4, no. 1, 2009.

[2] L. J. Akinbami, J. E. Moorman, and X. Liu, "Asthma prevalence, health care use, and mortality: United States, 2005-2009.," National health statistics reports, no. 32, pp. 1-14, Jan. 2011. 
[3] B. Mariani, M. C. Jimenez, F. J. G. Vingerhoets, and K. Aminian, "On-shoe wearable sensors for gait and turning assessment of patients with Parkinson's disease," IEEE Trans. Biomed. Eng., vol. 60, no. 1,pp. 155-158, Jan. 2013.

[4] B.-R. Chen et al."A web-based system for home monitoring of patients with Parkinson's disease using wearable sensors,'IEEE Trans. Biomed. Eng., vol. 58, no. 3, pp. 831-836, Mar. 2011.

[5] S. Patel et al., "Monitoring motor fluctuations in patients with Parkinson's disease using wearable sensors," IEEE Trans. Inf. Technol. Biomed., vol. 13, no. 6, pp. 864873, Nov. 2009.

[6] P. Castillejo, J. F. Martínez, J. Rodríguez-Molina, and A. Cuerva, "Integration of wearable devices in a wireless sensor network for an E-health application," IEEE Wireless Commun., vol. 20, no. 4, pp. 38-49, Aug. 2013.

[7] Deena M. Barakah \& Muhammad Ammad-uddin "Survey of Challenges and Applications of Wireless Body Area Network (WBAN) and Role of A Virtual Doctor Server in Existing Architecture" Third International Conference on Intelligent Systems Modelling and Simulation 2012

[8] Abdul Fuad Abdul Rahman, Rabiah Ahmad, Sofia Najwa Ramli "Forensics Readiness for Wireless Body Area Network (WBAN) System" February 16 19, 2014 ICACT2014.

[9] Minsoo Lee, LianhuaTian, Otgonjargal Myagmar "Design of a Particle Swarm Optimization-based

[10] Classification System in a WBAN Environment" International Journal of Advances in Computer Science and Technology (IJACST), Vol.2, No.8, Pages : 24-27 (2013) Special Issue of ICCECT 2013 - Held during 1617 August, 2013, ThailandBring Down Prices of Wearable Devices.[Online].Available: http://www.eetindia.co.in/ART_8800703467_1800007_ NT_c8cc7af5. HTM, accessed Sep. 28, 2014.

[11] L.Grossman and M. Vella, "iNeed?" TIME Mag., pp. 24-29, Sep. 2014.

[12] M. T. Quazi, "Human emotion recognition using smart sensors," M.S. thesis, School Eng. Adv. Technol., Massey Univ., Palmerston North, New Zealand, 2012.
[Online].Available:http://mro.massey.ac.nz/handle/10179 13364

[13] A. Bleicher, "Wearable computers will let us share thoughts and sensations," IEEE Spectra., pp. 66-71, Jun. 2014.

[14] Hassine Moungla, Nora Touati and Ahmed Mehaou, "A Power Harvesting, Dynamic and Reliable Wireless Body Area Networks Model Deployment for Health Care Applications", High Performance Computing and Simulation (HPCS), International Conference, IEEE 2012.

[15] Changhong Wang, Qiang Wang and Shunzhong Shi, "A Distributed Wireless Body Area Network for Medical Supervision", Instrumentation and Measurement Technology Conference (I2MTC), IEEE 2012.

[16] Kihyun Kim, Ick-Soo Lee, Mahnsuk Yoon, Junhyung Kim, Honggil Lee and Kijun Han, “An Efficient Routing Protocol based on Position Information in Mobile Wireless Body Area Sensor Networks", Networks and Communications, 2009, NETCOM'09, First International Conference IEEE 2009.

[17] N.K. Suryadevara and S. C. Mukhopadhyay,"Wireless sensor network based home monitoring system for wellness determination of elderly," IEEE Sensors J., vol. 12, no. 6, pp. 1965-1972, Jun. 2012.

[18] N. K. Suryadevara, A. Gaddam, R. K. Rayudu, and S. C. Mukhopadhyay, "Wireless sensors network based safe home to care elderly people: Behaviour detection," Sens. Actuators A, Phys., vol. 186, pp. 277-283, Oct. 2012.

[19] Review paper on "Wireless Body Area Network for Mobile Computing" National Conference on Recent Trends in Electronics \& Communication held at K. S. Institute of Technology, Bangalore on $30^{\text {th }}$ Aug 2013.

[20] M. M. Kermani, M. Zhang, A. Raghunathan, and N. K. Jha, "Emerging frontiers in embedded security," in Proc. 26th Int. Conf. VLSI Design 12th Int. Conf. Embedded Syst., Jun. 2013, pp. 203-208.

[21] C. Oliveira, M. Máckowiak, and L. M. Correia, "Challenges for body area networks concerning radio aspects," in Proc. 11th Eur. Wireless Conf., Vienna, Austria, Apr. 2011, pp. 1-5. 